The Egyptian Journal of Hospital Medicine (October 2019) Vol. 77 (5), Page 5534-5541

\title{
Epidemiological Typing of Methicillin Resistant Staphylococcus aureus Isolated from Surgical Site Infection Following Caesarean Section in an Egyptian University Hospital \\ Asmaa M. El-Nasser', Azza Hassan El Salakawy ${ }^{1}$, Azza Ahmed Mira ${ }^{1}$, Daad Fathallah Ibrahim ${ }^{1}$ and Hala Fathy El-Sharaky ${ }^{2}$ \\ ${ }^{1}$ Department of Medical Microbiology \& Immunology. ${ }^{2}$ Department of Obstetrics \& Gynecology, Faculty of Medicine for Girls, Al-Azhar University, Cairo, Egypt. \\ Corresponding author:Asmaa M. El-Nasser,email: asmalnasser_8080@ live.com,Mob: 01221567354
}

\begin{abstract}
Background: Surgical site infection (SSIs) is one of the most common healthcare-associated infections. It comprises a great risk to hospitalized patients in terms of morbidity, mortality and economic cost.

Objectives: To identify the causative organism(s) responsible for surgical site infection following Cesarean section and to trace the source of that infection.

Patients \& methods: A total of 623 clinical samples and swabs were collected from Obstetrics \& Gynecology Department at Al-Zahraa University Hospital. The study included 300 patients with provisional diagnosis of SSI from whom, wound swabs were taken, in addition to 23 swabs from medical staff and 300 environmental samples. Samples were subjected to conventional bacteriological identification, biochemical reactions, antibiogram and genotyping by Random Amplified Polymorphic DNA (RAPD) assay of the dominated isolates.

Results: Out of 300 wound swabs from patients with SSI following CS surgery, 62 (20.7\%) yielded aerobic microbial growth. Eighty three out of 323 swabs (26\%) collected from health care workers (HCWs) \& environment were positive on culture. The most common organism isolated from both sources (clinical \& environmental specimens) was Methicillin resistant Staphylococcus aureus (MRSA) isolates 35/623 (5.6\%). Genotyping of MRSA isolates revealed 20 distinct Random Amplified Polymorphic DNA (RAPD) patterns, indicating high rate of genetic heterogeneity among MRSA isolates.

Conclusion: There were clonally related MRSA isolates obtained from operating room and from patients, indicating that the operating room was accused as a source of infection.

Keywords: Surgical Site Infection, Methicillin-resistant Staphylococcus aureus, Random Amplified Polymorphic DNA.
\end{abstract}

\section{INTRODUCTION}

Surgical site infection remains the second most common adverse event occurring to hospitalized patients and a major source of morbidity following surgical procedures ${ }^{(1)}$. Surgical site infection is a type of health care associated infection in which wound infection occurs after an invasive or surgical procedure within 30 days postoperative ${ }^{(2)}$. There are many factors that are thought to affect the susceptibility of wound to infection including diabetes, systemic steroid use, extremes of age, poor nutritional status, prolonged pre-operative hospitalization and wound contamination ${ }^{(3)}$.

For most SSIs, the source of infection is the normal flora of the patient's skin, mucous membranes, or even the hollow viscera. Exogenous sources of SSI pathogens include surgical personnel, operating room environment including air, tools, and instruments. Exogenous flora is primarily aerobes, especially Grampositive organisms (e.g. staphylococci and streptococci) (4). Although the total elimination of wound infection is not possible, a reduction in the infection rate to a minimal level could have significant benefits in terms of both patient comfort and medical resources used.
Prevention of such infections is the responsibility of all individuals and services providing health care ${ }^{(5)}$.

Aim of the study: The study aimed to identify the most prevalent causative organism(s) responsible for surgical site infection following Cesarean section and to trace the source of that infection.

\section{PATIENTS AND METHODS}

Patients: The study included 300 female patients underwent Cesarean section. The patients were admitted to Al-Zahraa University Hospital with age ranged from 20 to 41 years (mean $27 \pm 4.6$ ) years old. They were randomly selected with clinically diagnosed SSI within 30 days post-operative, based on presence of at least one of the following criteria:

- Purulent discharge with or without laboratory confirmation.

- Organisms are identified from fluid or tissue by a culture or non-culture based microbiologic testing method

- At least one of the following symptoms \& signs: pain or tenderness, fever $\left(>38^{\circ} \mathrm{C}\right)$, localized swelling or redness at the site of surgical operation ${ }^{(6)}$. 
Patients who underwent other types of surgical operation, patients that stayed in the hospital for less than $24 \mathrm{~h}$ post-operatively or who did not have any apparent manifestations of SSI were excluded from the study.

The study also included 23 health-care workers (HCWs) from whom nasal and hand swabs were obtained. In addition, 300 environmental swabs were collected from different sources in the operating room.

\section{Ethical Consideration:}

The study was approved by the Institutional Human Ethical Committee of Faculty of Medicine, Al-Azhar University and all of the participants gave their informed written consent before collection of samples.

\section{Sample Collection and Processing}

Swab from the infected surgical wound from each target patient was obtained. Swabs from health care workers (HCWs) were collected from anterior nares of both sides, hands and throat. Environmental swabs were collected from different sources in the operating room (e.g instruments, lamps etc.). Swabs were placed into 5 $\mathrm{ml}$ of trypticase soy broth and transported immediately to the laboratory.

Swabs were inoculated onto sheep blood agar (5 $\%)$, MacConkey's agar and Nutrient agar plates and incubated at $37^{\circ} \mathrm{C}$ aerobically for $24-48$ hours. The obtained colonies were identified to genus level by conventional laboratory identification methods including: colonial morphology, Gram stain and biochemical reactions e.g. catalase test, coagulase test, indole test and Voges-Proskauer test.

Antimicrobial susceptibility test was carried out using the standardized disk diffusion method as described by Kirby-Bauer according to the CLSI, 2016 (7) (Clinical and Laboratory Standards institute, 2016). The following antimicrobials were tested: ampicillin, ofloxacin, amikacin and tetracycline. Oxacillin $(1 \mu \mathrm{g})$ and vancomycin discs were included for $S$. aureus. All isolated strains were stored on $10 \%$ glycerolsupplemented brain heart infusion (BHI) broth at $-20^{\circ} \mathrm{C}$ until required.

Genotyping by random amplified polymorphic DNA (RAPD) technique was applied upon MRSA isolates, the most likely organism prevalent in clinical specimens during study period ${ }^{(8)}$.

\section{DNA extraction:}

Genomic DNA from $S$. aureus isolates was prepared from Luria-Bertani broth cultures grown overnight at $37^{\circ} \mathrm{C}$ with aeration. Each culture was diluted ten-fold in $10 \mathrm{mM}$ Tris- $\mathrm{HCl}(\mathrm{pH} 8.0)$ and $1 \mathrm{mM}$ EDTA, boiled for $10 \mathrm{~min}$ and kept subsequently on ice. After centrifugation at $12000 \mathrm{rpm}$ for $3 \mathrm{~min}$, the resulting supernatant was used as the template for PCR assays ${ }^{(\mathbf{9})}$.

\section{RAPD-PCR assay:}

Three random primers were used for typing of MRSA isolates according to Byun et $\boldsymbol{a l} .{ }^{(8)}$ with sequence of: OPA-C05 (5'-GATGACCGCC-3'), OPA-C15 (5'GACGGATCAG-3') and OPA-F06 (5'GGGAATTCGG-3'). DNA was amplified in $25 \mu \mathrm{l}$ of a modified solution containing $12.5 \mu \mathrm{l}$ of Taq complete master mix (Alliance Bio- Canada) ( $15 \mathrm{mM}$ Tris$\mathrm{HCl}(\mathrm{pH} 8.3)$, four deoxynucleoside triphosphates, $100 \mu$ $\mathrm{M}$ each, $3 \mathrm{mM} \mathrm{MgCl} 2,1 \mathrm{U}$ of Taq polymerase, $3 \mu \mathrm{l}$ of random primer (Atlanta ,USA) (a single primer was used in each reaction) and $4.5 \mu \mathrm{l}$ of $\mathrm{dd}_{2} \mathrm{O}$. Finally, $5 \mu \mathrm{l}$ of DNA template was added to each $0.2 \mathrm{ml}$ reaction tube. Mixtures were subjected to 40 thermocycles of amplification $\left(94^{\circ} \mathrm{C}\right.$ for $30 \mathrm{sec}, 37^{\circ} \mathrm{C}$ for $1 \mathrm{~min}$ and 72 ${ }^{\circ} \mathrm{C}$ for $2 \mathrm{~min}$ for each cycle) in a thermal cycler (Biometra- Germany) to which we added one cycle in which the incubation at $72{ }^{\circ} \mathrm{C}$ lasted for $12 \mathrm{~min}$ and the first denaturation at $94{ }^{\circ} \mathrm{C}$ lasted for $5 \mathrm{~min}{ }^{(9)}$. The products of RAPD assay were separated by $2 \%$ agarose gel electrophoresis and were visualized after staining with ethidium bromide. Banding patterns were photographed using gel documentation system (Biometra- Germany). Positions of clearly scored RAPD fragments were transformed into a binary characters matrix (' 1 ' for the presence and ' 0 ' for the absence of a band at a particular position). Criteria established by Tenover et al. ${ }^{\left({ }^{9}\right)}$ were considered for genetic grouping and definition of the same genotypic strain.

\section{Statistical analysis}

Analysis was conducted for qualitative data using number, percent and Chi-square test $\left(\mathrm{x}^{2}\right)$. For numeric data, we used mean and standard deviation with significant level at $\mathrm{P}$ - value $<0.05$. Analysis of the results was performed using statistical software package SPSS version 17. Dendrogram was constructed from the percentage similarity values by software program of Unwighted Pair-Group Method of Arithmetic average (UPGMA) cluster analysis.

\section{RESULTS}

Out of 300 wound swabs from patients with SSI following CS surgery, 62 (20.7\%) yielded aerobic microbial growth, eight samples 8/300 (2.7\%) yielded mixed growth on culture, in which Coagulase negative staph (CoNS) and Proteus spp. was the commonest combination encountered in 2 samples. Fifty four samples out of $300(18 \%)$ yielded monomicrobial growth (Table 1). 
Table (1): Types \& frequency of organisms isolated from 300 female patients with clinically diagnosed SSI postCesarean section

\begin{tabular}{|c|c|c|}
\hline Type of growth & Isolated organisms & $\begin{array}{c}\text { No of } \\
\text { isolates }\end{array}$ \\
\hline $\begin{array}{l}\text { Monomicrobial growth: } \\
(\mathrm{n}=54,18 \%)\end{array}$ & $\begin{array}{l}\text { Gram positive isolates: } \\
\text { MRSA* }^{*} \\
\text { CoNS }^{* *} \\
\text { MSSA }^{* * *} \\
\text { Anthracoid bacilli } \\
\text { Candida }\end{array}$ & $\begin{array}{c}\mathbf{4 1}(\mathbf{1 3 . 7 \%}) \\
21 \\
7 \\
10 \\
2 \\
1\end{array}$ \\
\hline & $\begin{array}{l}\text { Gram negative isolates: } \\
\text { E.coli } \\
\text { Proteus spp. } \\
\text { Klebsiella spp. } \\
\text { Pseudomonas aeruginosa }\end{array}$ & $\begin{array}{c}\mathbf{1 3}(4.3 \%) \\
7 \\
1 \\
2 \\
3 \\
\end{array}$ \\
\hline Mixed growth $(n=8,2.7 \%)$ & Mixed & 8 \\
\hline No growth on culture $(n=238,79.3 \%)$ & & 238 \\
\hline Total & & 300 \\
\hline
\end{tabular}

MRSA $^{*} \quad$ Methicillin-resistant Staphylococcus aureus, CoNS ${ }^{* *}$ Coagulase negative staph, MSSA ${ }^{* * *}$ Methicillin-sensitive Staphylococcus aureus

The most common organism isolated from wound swabs was S. aureus 31/300(10.3\%), which was further identified according to susceptibility to oxacillin, revealing 10 MSSA isolates (3.3\%) with predominance of MRSA $21 / 300$ (7\%) among S. aureus isolates (Table 1). Eighty three out of 323 swabs (26\%) collected from health care workers (HCWs) \& environment were positive on culture. Eighteen isolates (18/323-5.6\%) were obtained from swabs of HCWs. Sixty five isolates were obtained from environmental samples (65/323 - 20\%), 37 of them were Gram positive organisms and 28 were Gram negative bacilli (Table 2). The most common organism isolated from both sources (clinical \& environmental specimens) was Methicillin resistant Staphylococcus aureus (MRSA) isolates $35 / 623(5.6 \%)$.

Table (2): Frequency and sources of pathogens isolated from HCWs and environment

\begin{tabular}{|l|l|c|}
\hline \multicolumn{1}{|c|}{ Source } & \multicolumn{1}{|c|}{ Isolates } & No \& \% of isolates \\
\hline $\begin{array}{c}\text { HCWs (total= 70) } \\
\text { Nasal swabs } \\
\text { Throat \& Hand }\end{array}$ & MRSA* & $3(0.9 \%)$ \\
CoNS** & $15(4.6 \%)$ \\
\hline $\begin{array}{l}\text { Environmental swabs of } \\
\text { operating room } \\
\text { (total=253) }\end{array}$ & Gram positive: (11.5\%) & \\
& MRSA & $11(3.4 \%)$ \\
& MSSA & $9(2.8 \%)$ \\
& CoNS & $8(2.5 \%)$ \\
& Diphteriods spp. & $5(1.5 \%)$ \\
& Enterococcus & $3(0.9 \%)$ \\
& Candida albicans & $1(0.3 \%)$ \\
& Gram negative: (8.7\%) & $6(1.9 \%)$ \\
& E.coli & $6(1.9 \%)$ \\
& Proteus spp. & $8(2.5 \%)$ \\
& Klebsiella spp. & $8(2.5 \%)$ \\
\hline No growth on culture & Pseudomonas aeruginosa & $240(74.3 \%)$ \\
\hline Total & & 323 \\
\hline
\end{tabular}

All tested $S$. aureus isolates that were resistant to oxacillin were also resistant to other antibiotics. However, all MRSA isolates were susceptible to vancomycin except only one environmental isolate. Resistance to oxacillin, trimethoprim/ sulfamethoxazole \& gentamicin was detected in 27 (33.8\%), 18(22.5\%) \& 11(13.8\%) clinical isolates respectively. The same resistance profile was also detected in 46 (23.7\%), 35 (18\%) \& 23 (11.9\%) environmental isolates respectively with statistical significant difference between both sources of isolates as regard antibiotic resistance $(\mathrm{P}<0.05)$. 
RAPD-PCR was employed on 35 MRSA isolates using 3 different primers. The primers yielded 32 bands, 29 of them were polymorphic with sizes ranging between 75 up to $570 \mathrm{bp}$. Up to 3 bands were observed in each fingerprint (common band) with sizes ranging between $75 \mathrm{bp}$ and $120 \mathrm{bp}$ molecular size and 16 bands were unique (isolate specific marker). Thus 32 distinct bands were obtained and used for cluster analysis (Fig 1, 2 \& 3).

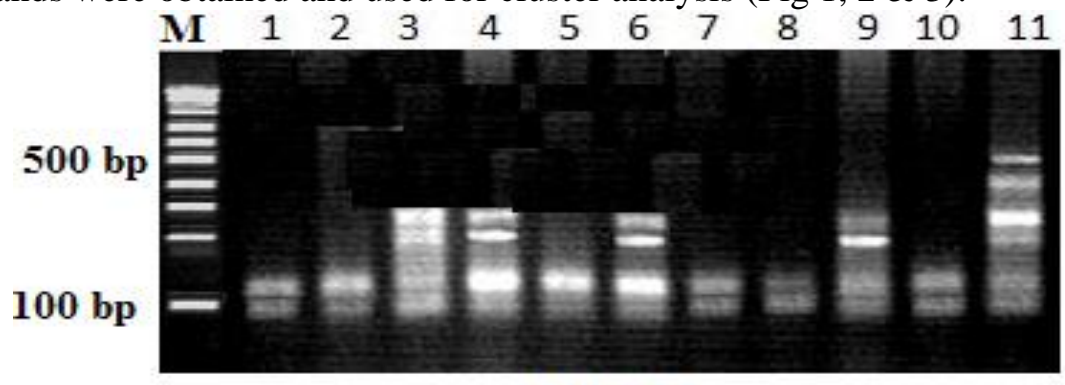

OP-C15

Fig. (1): 2\% agarose gel of RAPD-PCR patterns generated from eleven MRSA isolates (lanes 1- 11) using primer OP-C15, M; 100bp ladder DNA marker

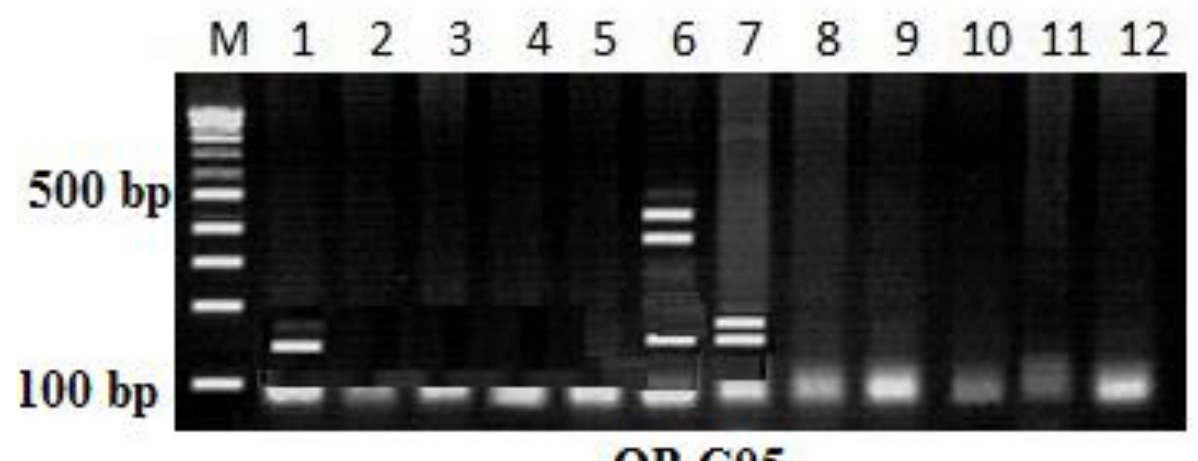

\section{OP-C05}

Fig. (2): 2\% agarose gel of RAPD-PCR patterns generated from twelve MRSA isolates primer OP-CO5.

(lanes 1-12) using

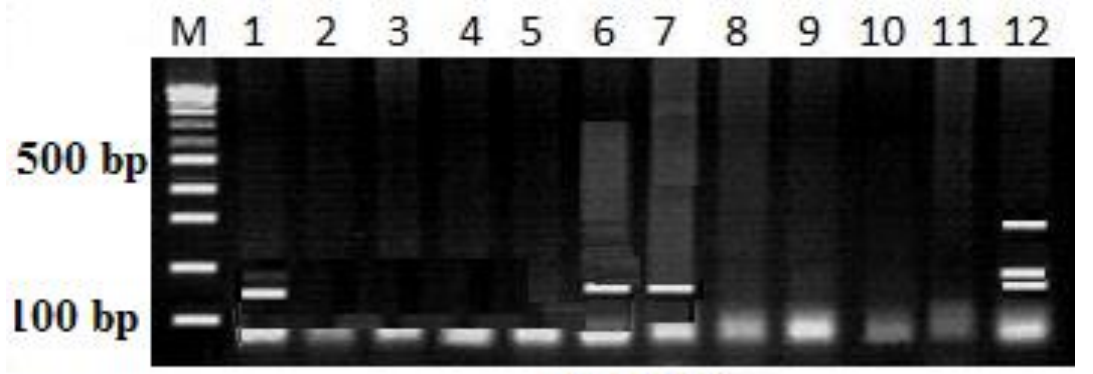

OP-F06

Fig. (3): 2\% agarose gel of RAPD-PCR patterns generated from twelve MRSA isolates (lanes 1- 12) using primer OP-F06.

Cluster analysis grouped all isolates into 3 major clusters. The 3 major clusters included 20 distinct RAPD patterns at $100 \%$ similarity level (Fig 4). 


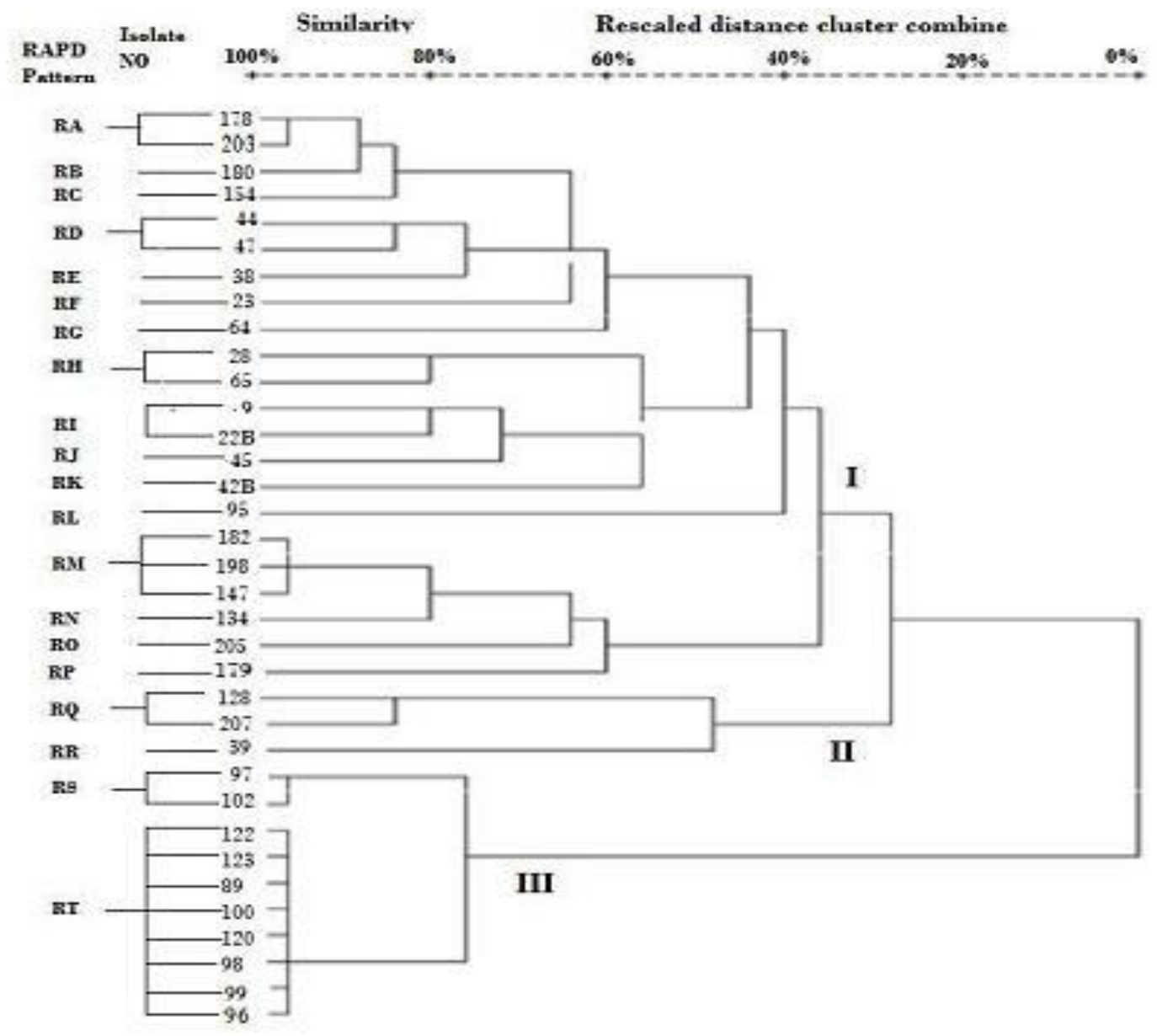

Fig. (4) Dendrogram estimated twenty RAPD patterns (A-T) by using the results of RAPD assays with 3 primers among 35 MRSA isolates

\section{DISCUSSION}

Surgical site infection (SSI) continues to be one of the most common complications in surgical units with significant increased costs, morbidity and potential mortality ${ }^{(\mathbf{1 0})}$. There is a wide variability of incidence of SSI following CS in different countries and in various localities in the same country. Our study showed a SSI rate of $20.7 \%$ among 300 patients underwent Cesarean section. In USA, an incidence of SSI post CS of $6.5 \%$ was reported ${ }^{(11)}$. While in African countries, the incidence was $10.9 \%$ in Tanzania ${ }^{(12)}$, 9.1\% in Nigeria ${ }^{(\mathbf{1 3})}$. A study from Estonia reported a SSI rate of $6.2 \%$ among 305 patients that underwent Cesarean section and had a 30 days follow up postsurgery. A cohort of women with Cesarean section from Thai- Myanmar border showed a SSI rate of $5.9 \%$. An Italian study reported a SSI rate of $4.7 \%$ from 430 mothers with Cesarean section. All the above studies reported a lower rate of SSI among patients that underwent Cesarean section as compared to our study. The variability of reported incidence of SSI could be attributed to many risk factors of SSI as the operative time, the duration of post-operative hospital stay, the dosing \& timing of prophylactic antibiotics, different maternal age, the different hygienic standards and surveillance methods used to identify infections.

In our study, Gram positive organisms were dominant from both infected wounds and environmental samples constituted $13.7 \%$ (41/300), $11.5 \%$ (37/323) respectively, which can be attributed to an outbreak of Gram positive pathogens in the hospital environment during the period of sample collection. This comes in concordance with Tang and Wan ${ }^{(14)}$ who found in their study that in the operating theatre area, the isolation rates of Gram positive bacteria exceeded those of Gram negative bacteria. In addition, Shin et al. ${ }^{(5)}$ proved that operating rooms environment contained a microbial community similar to the one found on human skin, dominated by Staphylococcus \& Corynebacterium indicating that operating rooms are dominated by humans associated microbiota. On the other hand, this finding is in contrast with the result found by Saini et al. ${ }^{(15)}$ who 
reported that Gram negative bacilli were the principle colonizers in the hospital environment. Also our study disagrees with the data recorded by Sohn et al. ${ }^{(16)}$ who reported the predominance of Gram negative bacilli as the etiology of SSI in different types of surgeries. This finding may be interpreted by the type and anatomical site of surgery in this study. In abdominal surgeries, there is an increased risk for Gram negative bacilli and other coliforms to be causative agents of wound infection. In addition, the variation in the spectrum of SSI causative organisms as stated by Kathryn et al. ${ }^{(13)}$ may be due to the fact that prophylactic antibiotics though effective, yet may fail when they are used inappropriately or when the wrong one is used.

In our study, the most common organism isolated from patients with SSI was S.aureus 31/300 (10.3\%). This result comes in agreement with those obtained by Jido and Garba ${ }^{(12)}$, Kathryn et al. ${ }^{(13)}$ and Saini et al. ${ }^{(15)}$ with $S$. aureus percentages of $31.8 \%, 31.27 \%$ and $27.3 \%$ respectively. This may be attributed to the fact that the anatomy of the female genital system exposes her to contamination by $S$. aureus endogenously colonizing the vagina of healthy women ${ }^{(17)}$. S. aureus isolated from clinical specimens was further identified according to susceptibility to methicillin, revealing 10 MSSA isolates (3.3\%), with predominance of MRSA $(\mathrm{n}=21,7 \%)$ among $S$. aureus isolates. This finding is consistent with the of Jama $\boldsymbol{e t}$ al. ${ }^{(18)}$, Warner and Onderdonk ${ }^{(19)}$ and Nisa et al. (20).

In our study, screening of HCWs for bacterial colonization was performed. This screening revealed predominance of CoNS in samples obtained from throat and hands (15 out of 23 swabs), also HCWs were found to be nasally colonized by MRSA ( 3 out of 23 swabs). All MRSA isolates were sensitive to vancomycin. This finding is in agreement with the result obtained in Egyptian study by Alaa Eldeen $\boldsymbol{e t}$ al. (21) which revealed MRSA nasal carriage rate of (2.3\%) [two out of 84] among medical residents in Gynecology department of Al-Azhar University Hospital, Damietta branch. Also, our study is in agreement with study conducted by Geoffrey ${ }^{(22)}$ who reported MRSA colonization rate of $2.1 \%$ in the nasal cavity and axilla of HCWs. On the other hand, Costa et al. (23) found that $25.5 \%$ of HCWs in a Brazilian hospital were colonized by CoNS in throat, $14 \%$ were colonized by $S$. aureus and $6.7 \%$ were colonized by both. MRSA was not detected and all isolates were sensitive to ciprofloxacin and linezolid.
Transmission of pathogens within the hospital environment remains a hazard for hospitalized patients. Cross transmission of micro-organisms from inanimate surfaces may have a significant role in SSI. In our study, Sixty five isolates were obtained from environmental samples (20\%), 37 of them were Gram positive organisms and 28 were Gram negative bacilli. Our results revealed a lower rate of environmental contamination compared to the results conducted by Brady et al. ${ }^{(24)}$ who reported $41.4 \%$ of environmental samples contamination from bed handsets of surgical ward. These samples revealed pathogens known to cause SSI including MRSA (12.9\%), MSSA (2.9\%) and coliforms $(2.9 \%)$.

However, a higher rate of environmental contamination by MRSA (12.9\%) was reported by Brady et al. ${ }^{\text {(24) }}$ compared to our study, where MRSA contamination of operating room environment represented $(\mathrm{n}=11,4.3 \%)$. The high rates of MRSA contamination are likely to be a reflection of both poor hand hygiene practices and compromised hygienic measures. In this concern, it was found that prolonged operating time and increased duration of hospital stay preoperatively were risk factors for increased incidence of SSI ${ }^{(25)}$. MRSA remains one of the most important threats to public health due to the fast dissemination and fast diversification of MRSA strains with greater virulence and anti-microbial resistance ${ }^{(6)}$.

In the present study, a total of 32 distinct bands were used for cluster analysis of 35 MRSA isolates which were classified into 3 major cluster groups (I, II, III), defined at $70 \%$ similarity level. The majority of MRSA isolates belonged to cluster I ( 22 isolates), the other isolates were distributed as follows: 3 isolates in cluster II and 10 isolates in cluster III.

Our results are comparable with those obtained by Romanelli et al. (26), who studied 11 MRSA isolates by RAPD amplification, using 3 different primers $(1 \mathrm{~A}, 1 \mathrm{~B}, 1 \mathrm{C})$. Cluster analysis grouped all isolates into 3 major clusters at a similarity level $<60 \%$. The 3 major groups revealed 7 distinct RAPD patterns. A phylogenetic study demonstrated $90 \%$ of similarity among MRSA isolates with acceptable discriminatory power.

In the current study, it was observed that among the OP-C 15 generated amplicons, one amplicon of molecular size $120 \mathrm{bp}$ was shared by almost all studied MRSA isolates. Similarly, with OP$\mathrm{C} 05$, one band of molecular size $95 \mathrm{bp}$ was common to nearly all isolates and one band of molecular size 75 bp was shared by almost all MRSA isolates with OP- 
F06. The sharing of common bands indicated the presence of a highly conserved genomic region in MRSA isolates and at the same time confirmed the conventional bacterial identification of this organism. In addition, the present study clonally related isolates from the environment and from patients, indicating transmission of infection between both of them.

Our results match those of the study conducted by Shehata (27) in which amplification reactions generated up to 105 bands, 50 of them were polymorphic with sizes ranging between 200 to 2500 bp. Up to 9 bands were observed in all MRSA isolates. Isolate Sc 7 presented a unique band, this band could be used to characterize and identify it. This study approved that RAPD-PCR technique is suitable for typing both within and between bacterial species.

\section{CONCLUSION}

There is a high incidence of post CS-SSI due to MRSA, which still represents a real risk of surgery. Thus, a comprehensive infection control strategy based on hand hygiene, education and training in antibiotic prescribing, environmental cleaning, contact precautions, good antibiotic stewardship and an active surveillance system on the basis of international criteria is urgently needed. There were clonally related MRSA isolates obtained from operating room and from patients, indicating that the operating room was accused as a source of infection.

- Genotyping by RAPD-PCR is a promising, simple, rapid and helpful tool for routine epidemiological surveillance of nosocomial infection and cost effective for monitoring application of infection control measures at health care facilities.

\section{REFERENCES}

1. Shree R, Park SY, Beigi RH, Dunn SL, Krans EE (2016): Surgical Site Infection following Cesarean Delivery: Patient, Provider, and Procedure-Specific Risk Factors. Am J Perinatol., 33 (2): 157-64.

2. Haun W, Higgins A, London J, Charland S, Ratzer E and Clark J (2016): Are prophylactic antibiotics necessary in elective laparoscopic cholecystectomy, regardless of patient risk? Ann Surg Treat Res., 93 (2): 76-81.

3. Ezechi OC, Edet A, Akinlade H, Gab-Okafor CV, Herbertson E (2009): Incidence and risk factors for Caesarean wound infection in Lagos Nigeria. BMC., 2: 186194.

4. Shin MH, Rosen AK, Chen Q, O'Brien W, Shwartz M, Mull HJ (2015): The effects of local warming on surgical site infection. Surg Infect., 6: 595-603.

5. Valle A, Daniel D, ErdanK M, Della J (2016): Hypoalbuminemia Independently Predicts Surgical Site
Infection, Pneumonia, Length of Stay, and Readmission After Total Joint Arthroplasty; J Arthroplasty, 31: 15-21.

6. Centers for Disease Control, Prevention CDC (2008): CDC definition of health care- associated infection and criteria for specific types of infections in the acute care setting. Am. J. Infect. Control, 36: 309-332.

7. Clinical and Laboratory Standards Institute (2016): Performance Standards for Antimicrobial Susceptibility Testing. 29th ed. CLSI supplement M100. Wayne, PA: Clinical and Laboratory Standards Institute.

8. Byun, DE, Kim SH, Shin JH, Suh SP, Ryang DW (1997): Molecular epidemiologic analysis of Staphylococcus aureus isolated from clinical specimens. J. K. Med. Sci., 12: 190198.

9. Tenover FC, Arbeit RD, Goering RV (1997): How to select and interpret molecular strain typing methods for epidemiological studies of bacterial infections: A review for health care epidemiologists. Infect. Control Hosp. Epidemiol., 18: 426-439.

10. Centers for Disease Control and Prevention CDC (2004): Office of biosafety, hospital infections program. Recommendations on infective waste, Atlanta, GA: Department of Health and Human Services: 1-6. https://www.cdc.gov/labs/pdf/CDC-

BiosafetyMicrobiologicalBiomedicalLaboratories-2009P.PDF

11. Mpogoro FJ, Mshana SE, Mirambo M, Kidenya B, Gumodoka B (2014): Incidence and predictors of surgical site infections following caesarean sections at Bugando Medical Centre .Antimicrob Resist Infect Control, 11: 3-25.

12. Jido TA, Garba ID (2012): Surgical-site infection following cesarean section in Kano, Nigeria. Ann Med Health Sci Res., 2 (1): 33-36.

13.Kathryn C K, Maine R, Trelles M (2015): Cesarean Section Surgical Site Infections in Sub-Saharan Africa: A Multi-Country Study from Medecins Sans Frontieres. World J Surg., 39: 350-355.

14. Tang R, Wang YL (2013): Surgical site infection in pancreaticoduodenectomy: Does the SCIP-approved regimen provide adequate coverage? Surgery, 154 (2): 190196.

15.Saini S., Gupta N., Aparna, Lokveer and Griwan MS (2004): Surgical infections: A microbiological study. J. Appl. Microbiol., 8: 118-125.

16. Sohn AH, Parvez FM, Hai HH, Bich NN, Thanh NH, Viet TV, Archibald LK (2002): Prevalence of surgical site infections and patterns of antimicrobial use in a large tertiary care hospital in Ho Chi Minh City, Vietnam. J. Infect. Control Hosp. Epidemiol., 23: 382-387.

17. Dhar H, Rathi B, Al- Busaidi I, Hamdi I (2014): A Study of Post-Caesarean Section Wound Infections in a Regional Referral Hospital, Oman. Sultan Qaboos University Med J., 14: 211-217

18.Jama S, Bakker O, Van santvoort H (2012): Endoscopic Transgastric vs Surgical Necrosectomy for Infected Necrotizing PancreatitisA Randomized Trial. JAMA., 307: 1053-1065. 


\section{ejhm.journals.ekb.eg}

19. Warner J, Onderdonk B (2004): Diversity of toxic shock syndrome toxin-positive Staphylococcus aureus isolates. Appl. Environ. Microbiol., 70: 6931-6935.

20.Nisa M, Naz T, Afzal I, Hassan L (2005): Scope of surgical site infection in Obstetrics and Gynecology. JPMI., 19: 438-441.

21. Alaa Eldeen M H, Ali I , Khaled E, Mohammed A A, Hany A, Ahmed A E, Fathia M E (2018): Nasal colonization of methicillin-resistant Staphylococcus aureus among medical residents in Al-Azhar University Hospital, Damietta branch. Al-Azhar Assiut Medical Journal, 16: 8795.

22. Geoffrey W, Neil N, Michael A, David B, Daniel J (2015): Cost Analysis and Surgical Site Infection Rates in Total Knee Arthroplasty Comparing Traditional vs. Single-Use Instrumentation. J Arthroplasty, 30: 2271-2274.
23. Costa AM, Lotfi CJ, de Cavalcanti RC (2014): The time course and microbiology of surgical site infections after head and neck free flap surgery. Laryngoscope, 125: 10841089.

24. Brady RR, Kalima P, Damani NN, Wilson RG, Dunlop MG (2007): Bacterial contamination of hospital bed-control handsets in a surgical settings: A potential marker of contamination of the healthcare environment. Ann. R. Coll. Surg. Engl., 89: 656-660.

25. Iyamba J, Wambale J, Lukukula C, Takaisi-Kikuni $\mathbf{N}$ (2014): High prevalence of methicillin resistant staphylococci strains isolated from surgical site infections in Kinshasa. Pan Afr Med J., 18: 322-4440.

26. Romanelli RM, Clement WT, Lima SS, Rezende EM, Martinho GH (2010): MRSA outbreak at a transplantation unit. Braz.J. Infect. Dis., 14: 54-59.

27. Shehata AI (2008): Phylogenetic diversity of Staphylococcus aureus by random amplification of polymorphic DNA. Aust. J. Basic \& Appl., 2: 858-863. 